\title{
MicroRNA-425-5p Is Involved in the Development of Diabetic Retinopathy and Regulates the Proliferation and Migration of Retinal Microvascular Endothelial Cells
}

\author{
Xia Liu ${ }^{\mathrm{a}}$ Yanhua Zhou ${ }^{\mathrm{b}}$ Yunxia Liu ${ }^{\mathrm{c}}$ Qian Wang ${ }^{\mathrm{a}}$ Lili Pan $^{\mathrm{a}}$ \\ ${ }^{a}$ Department of Ophthalmology, Affiliated Hospital of Weifang Medical University, Weifang, China; ${ }^{b}$ Department \\ of Oncology, Affiliated Hospital of Weifang Medical University, Weifang, China; 'Department of Internal Medicine, \\ Fuyanshan Branch of Affiliated Hospital of Weifang Medical University, Weifang, China
}

\section{Keywords}

Diabetic retinopathy $\cdot$ MicroRNA-425-5p · Diabetes mellitus ·

Diagnosis · Vascular endothelial cell

\begin{abstract}
Introduction: The objective of this article was to detect the expression pattern and clinical value of miR-425-5p in diabetic retinopathy (DR) patients and investigate its effect on the proliferation and migration of human retinal microvascular endothelial cells (HRMECs) in a high glucose (HG) state. Methods: The serum miR-425-5p level of the subjects was determined using quantitative real-time PCR. The diagnostic value of serum miR-425-5p was validated using the receiver operating characteristic curve. Pearson analysis detected the correlation between clinical indicators and microRNA. The influence of miR-425-5p on cell proliferation and migration under HG conditions was calculated by using Cell Counting Kit-8 and Transwell assay. Results: Serum miR-425-5p levels showed a gradual increasing trend in the healthy control group, the diabetic mellitus patients without DR, and DR patients. Moreover, the levels of miR-425-5p in proliferative DR (PDR) patients were elevated than that of non-PDR (NPDR) patients. Furthermore, upregulated miR-425-5p had a high diagnostic value for DR patients and can distinguish
\end{abstract}

karger@karger.com www.karger.com/ore

Karger!
(C) 2021 The Author(s)

Published by S. Karger AG, Basel

This is an Open Access article licensed under the Creative Commons Attribution-NonCommercial-4.0 International License (CC BY-NC) (http://www.karger.com/Services/OpenAccessLicense), applicable to the online version of the article only. Usage and distribution for commercial purposes requires written permission.
PDR patients from NPDR patients. The expression of miR425-5p was significantly positively correlated with the fasting plasma glucose, glycosylated hemoglobin ( $\mathrm{HbA1C})$, homeostasis model assessment of insulin resistance, and disease course of the patients. Under HG conditions, overexpression of miR-425-5p promoted HRMEC proliferation and migration, while inhibition of miR-425-5p led to opposite results. Conclusion: Present research confirmed that serum miR-425-5p levels in DR are marked by elevation. High expression of miR-425-5p can be used as a feasible diagnostic biomarker for DR patients and can predict the development and severity of DR. Moreover, inhibiting the expression of miR-425-5p levels under the condition of hyperglycemia may be used as a valuable therapeutic strategy for preventing the pathogenesis of DR.

(C) 2021 The Author(s)

Published by S. Karger AG, Basel

\section{Introduction}

Microvascular complications of diabetic mellitus (DM) include diabetic retinopathy (DR) [1]. The prevalence of DR in DM patients is about $1 / 3$, and $1 / 10$ of them have a threat to normal visual status [2]. Pathologically, DR damages the retinal microvascular system, capillary 
permeability, and retinal blood barrier and further leads to macular edema, retinal osmosis, vitreous hemorrhage of the retina, angiogenesis, and retinal detachment, thereby seriously damaging vision [3]. According to the early generation of new blood vessels, DR is usually classified into 2 different severity levels: divided into nonproliferative DR (NPDR) and proliferative DR (PDR). The formation of proliferation neovascularization for PDR patients will lead to vitreous hemorrhage and complications such as traction retinal detachment, resulting in irreversible vision loss $[4,5]$. Therefore, understanding the formation and regulation of retinal blood vessels is helpful to improve the treatment of this disease. At the same time, the disease is asymptomatic before significant pathological progress, and the current effective treatment of DR is only applicable to the late stage of the disease with significant adverse reactions [6]. Therefore, biological diagnostic markers involved in disease progression are of great significance for the treatment of DR.

Abnormal expression of microRNAs (miRNAs) is associated with the pathogenesis and pathological process of various human diseases [7]. It is proved that miRNA levels have a major influence on DM and its complications including DR. For example, miR-18a-5p affects DR and inhibits retinal angiogenesis by targeting fibroblast growth factor 1 and hypoxia-inducible factor 1-alpha [8]. miR-590-3p inhibits the apoptosis of DR cells by targeting the NLR family pyrin domain-containing 1 and inactivating the NADPH oxidase 4 signaling pathway [9]. It is noteworthy that miR-425-5p is identified to be aberrantly expressed in DR patients and nondiabetic control groups by microarray analysis [10]. miR-425-5p is also reported to be involved in the development of DM. Additionally, Luo et al. [11] have found that the NF- $\kappa \mathrm{B} / \mathrm{miR}$ $425-5 p / M C T ~ 4$ axis activates high glucose (HG) and IL$1 \beta$-induced endothelial cell injury in DM. However, the specific role of miR-425-5p on the progress of DR has not been introduced in detail.

The present research was to verify the expression level of miR-425-5p in DR and evaluate its clinical significance in the diagnosis and prediction of severity of DR. Besides, the regulatory role of $\mathrm{miR}-425-5 \mathrm{p}$ in the proliferation and migration of human retinal microvascular endothelial cells (HRMECs) under HG was also examined.

\section{Materials and Methods}

Study Participants and Clinical Samples

A total of 135 types 2 diabetic patients and 60 age- and gendermatched healthy volunteers, who visited the Affiliated Hospital of
Weifang Medical University from June 2015 to December 2017, were recruited. The inclusion criteria for the type 2 diabetic patients were as follows: (1) all patients met the criteria of the American Diabetes Association [12, 13]; (2) age above 18 years; (3) continuous subcutaneous insulin infusion or multiple insulin injections per day. Patients with acute complications such as diabetic ketosis, cardiovascular and cerebrovascular disease events, trauma surgery, acute and chronic infections, liver diseases, and other endocrine and metabolic diseases were excluded. All participants underwent ophthalmology examinations and based on the results of their fundus fluorescein angiography, and all diabetics were divided into 3 groups: 35 DM patients without DR (NDR group); 55 DM patients with NPDR; and 45 DM patients with PDR. The diagnostic criteria of DR are determined by 2 ophthalmologists independently according to the standards of the International Diabetic Retinopathy Project Guidelines [14]. Approximately $10 \mathrm{~mL}$ of whole blood was drawn from the elbow veins of all participants, serum samples were centrifuged, and stored at $-80^{\circ} \mathrm{C}$ until use. The patient's age, gender, BMI, and course of the disease were recorded, and the clinical characteristics of the subjects, such as total cholesterol (TC), triacylglycerol (TG), fasting plasma glucose (FPG), glycosylated hemoglobin (HbA1c), fasting insulin, and the calculated homeostasis model assessment of insulin resistance (HOMA-IR) were routinely detected. All the data are given in Table 1 .

\section{Ethics}

The research methods meet the standards set out in the Helsinki Declaration. The project was authorized by the Research Ethics Committee of the Affiliated Hospital of Weifang Medical University (No. 2015-097). All participants were informed of the purpose of the study and experimental procedures and signed a consent form approved by the Ethics Committee before participating.

\section{Cell Culture, Transfection, and Treatment}

HRMECs were obtained from Bena Culture collection (Beijing, China) and cultured in endothelial cell medium containing $10 \%$ fetal bovine serum and incubated in a constant temperature incubator at $37^{\circ} \mathrm{C}$ and $5 \% \mathrm{CO}_{2}$. According to previous studies, in vitro DR model was established through glucose induction $[15,16]$, HRMECs were cultured in a hyperglycemia medium containing 25 $\mathrm{mM}$ glucose (HG group), while the glucose control (low glucose [LG] group) cells were cultured in an HG medium containing 5 $\mathrm{mM}$. Osmotic control group cells were cultured in $5 \mathrm{~mm}$ glucose and $20 \mathrm{~mm}$ mannitol to maintain osmotic pressure balance. Meanwhile, to regulate the expression level of $\mathrm{miR}-425-5 \mathrm{p}$ in vitro, $\mathrm{miR}$ 425-5p mimics, miR-425-5p inhibitors, and negative controls (mimic NC and inhibitor NC) were transfected at the logarithmic growth stage of cells. Lipofectamine 2000 (Invitrogen, Carlsbad, CA, USA) was used as the cell transfection reagent, and the new medium was replaced $6 \mathrm{~h}$ after transfection. For transfected cells, the DR model was established by adding glucose $24 \mathrm{~h}$ after transfection.

Total RNA Extraction and Quantitative Real-Time PCR Assay

RNA in serum samples and HRMECs were extracted by TRIzol reagent. Isolated RNA was reverse transcribed into complementary DNA using the PrimeScript RT Master Mix Kit (Takara, Dalian, China). Furthermore, the quantitative real-time PCR assays 
Table 1. Comparison of general data and clinical data of each group

\begin{tabular}{lllll}
\hline Indicator & $\begin{array}{l}\text { Healthy control } \\
(n=60)\end{array}$ & $\begin{array}{l}\text { NDR } \\
(n=35)\end{array}$ & $\begin{array}{l}\text { NPDR } \\
(n=55)\end{array}$ & $\begin{array}{l}\text { PDR } \\
(n=45)\end{array}$ \\
\hline Age, years & $50.01 \pm 0.53$ & $50.14 \pm 0.64$ & $50.00 \pm 0.73$ & $50.25 \pm 1.03$ \\
Gender, male/female & $31 / 29$ & $21 / 14$ & $30 / 25$ & $25 / 20$ \\
BMI, kg/m 2 & $22.11 \pm 1.18$ & $21.80 \pm 1.19$ & $22.03 \pm 1.29$ & $21.72 \pm 1.14$ \\
Disease course, year & - & $3.92 \pm 7.11$ & $12.74 \pm 1.65$ & $13.70 \pm 1.74$ \\
TC, mmol/L & $4.43 \pm 0.35$ & $4.36 \pm 0.55$ & $4.60 \pm 0.57$ & $4.58 \pm 0.58$ \\
TG, mmol/L & $1.50 \pm 0.25$ & $1.51 \pm 0.15$ & $1.54 \pm 0.19$ & $1.49 \pm 0.29$ \\
FPG, mmol/L & $4.90 \pm 0.41$ & $5.41 \pm 0.40^{*}$ & $6.90 \pm 0.56^{*, \#}$ & $7.21 \pm 0.62^{*, \#, \&}$ \\
HbA1c, \% & $4.35 \pm 0.20$ & $6.28 \pm 0.36^{*}$ & $7.21 \pm 0.56^{*, \#}$ & $7.93 \pm 0.65^{*, \#, \&}$ \\
HOMA-IR & $1.28 \pm 0.048$ & $1.54 \pm 0.10^{*}$ & $1.65 \pm 0.17^{*, \#}$ & $1.82 \pm 0.19^{*, \#, \&}$ \\
FINS, mIU/L & $5.34 \pm 0.33$ & $5.75 \pm 0.15^{*}$ & $5.49 \pm 0.44^{*, \#}$ & $5.56 \pm 0.38^{*, \#}$ \\
\hline
\end{tabular}

TC, total cholesterol; TG, triacylglycerol; FPG, fasting plasma glucose; HbA1c, glycosylated hemoglobin; HOMA-IR, homeostasis model assessment of insulin resistance; FINS, fasting insulin; DR, diabetic retinopathy; NDR, diabetic mellitus patients without DR; PDR, proliferative DR; NPDR, non-PDR. ${ }^{*} p<0.05$, compared with healthy control group. ${ }^{\#} p<0.05$, compared with NDR, group. ${ }^{\&} p<0.05$, compared with NPDR, group. were performed on ABI7300 (Applied Biosystems, Foster City, CA, USA) thermal circulator using the SYBR Mixture and the ROX assay kit (CWBiotech, Beijing, China). U6 was employed as an internal standard; the relative levels of miR-425-5p were calculated according to the $2^{-\Delta \Delta C t}$ method. The primer sequences used in this study were: miR-425-5p forward: $5^{\prime}$-GGGGAGTTAGGATTAGGTC-3', reverse: 5'-TGCGTGTCGTGGAGTC-3"; U6 forward $5^{\prime}$-AACGCTTCACGAATTTGCGT- $3^{\prime}$ and reverse $5^{\prime}$-CTCGTTCGGCAGCACA-3". The quantitative real-time PCR reaction procedure was $95^{\circ} \mathrm{C}, 30 \mathrm{~s}$, and then 40 cycles of $95^{\circ} \mathrm{C}$ for $15 \mathrm{~s}, 60^{\circ} \mathrm{C}$ for $30 \mathrm{~s}$. Each experiment values represent 3 biological replicates.

\section{Cell Proliferation Assay}

Cell proliferation was detected by using Cell Counting Kit-8 assay; $5 \times 10^{3}$ HRMECs cells in the logarithmic growth phase were inoculated into each well of the 96-well plate. The cell viability was detected at $0,24,48$, and $72 \mathrm{~h}$, respectively. Before detection, $10 \mu \mathrm{L}$ Cell Counting Kit-8 (Beyotime, Shanghai, China) reagent was supplemented. After 2-h incubation, optical density was assessed at $450 \mathrm{~nm}$ using an iMark Microplate Absorbance Reader (Bio-Rad Laboratories, Hercules, CA, USA).

\section{Transwell Assay}

Transwell migration assay was used to detect the motility of cells. HRMECs were seeded at $2.5 \times 10^{3}$ cells/well into the upper chamber for serum-free culture, and the lower chamber was added with a medium containing $10 \%$ serum. After $24 \mathrm{~h}$, the unmigrated cells were swabbed with a cotton swab. The migrated cells were fixed with paraformaldehyde and stained with crystal violet for $20 \mathrm{~min}$. The number of cells in 5 fields was counted.

\section{Statistical Analysis}

All data in the study were statistically analyzed by using GraphPad Prism 7.0 software and SPSS 21.0 software and shown as mean \pm standard deviation. Student's test and one-way ANOVA fol-

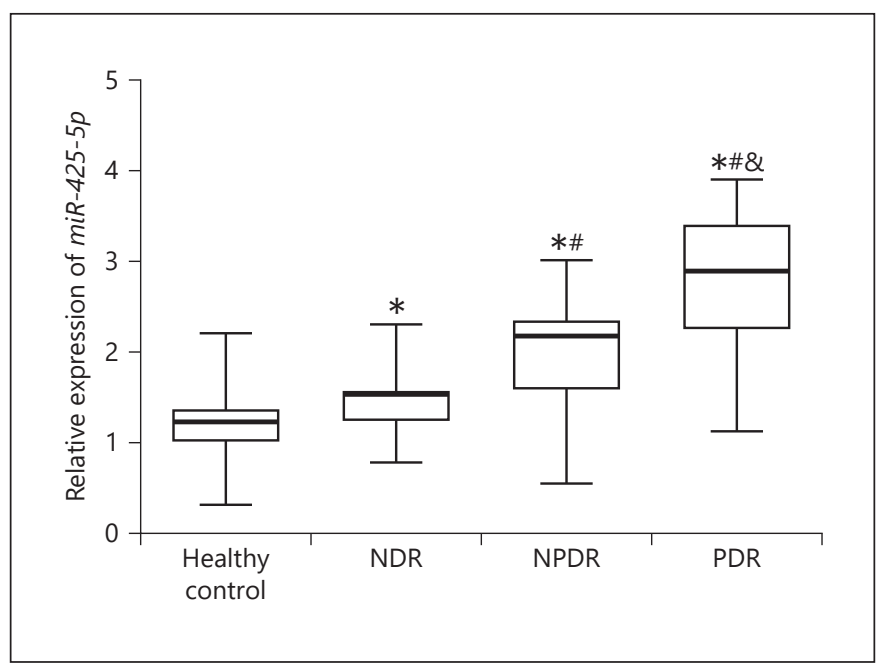

Fig. 1. Expression level of miR-425-5p in different groups was detected by RT-qPCR. Compared with the healthy control group and NDR patients, the expression level of miR-425-5p in DR patients was significantly increased, and PDR patients were significantly higher than NPDR patients. ${ }^{*} p<0.05$, compared with healthy control group; ${ }^{*} p<0.05$, compared with NDR group; and $p<0.05$, compared with NPDR group. DR, diabetic retinopathy; NDR, diabetic mellitus patients without DR; PDR, proliferative DR; NPDR, non-PDR; RT-qPCR, quantitative real-time PCR.

lowed by post hoc Tukey's test analysis were detected statistical differences between groups. Pearson analysis detected the correlation between clinical indicators and miRNA levels. The diagnostic value of serum miR-425-5p in patients was evaluated by the receiver operating characteristic (ROC) curve. Differences were considered to be significant at $p<0.05$.
Liu/Zhou/Liu/Wang/Pan 


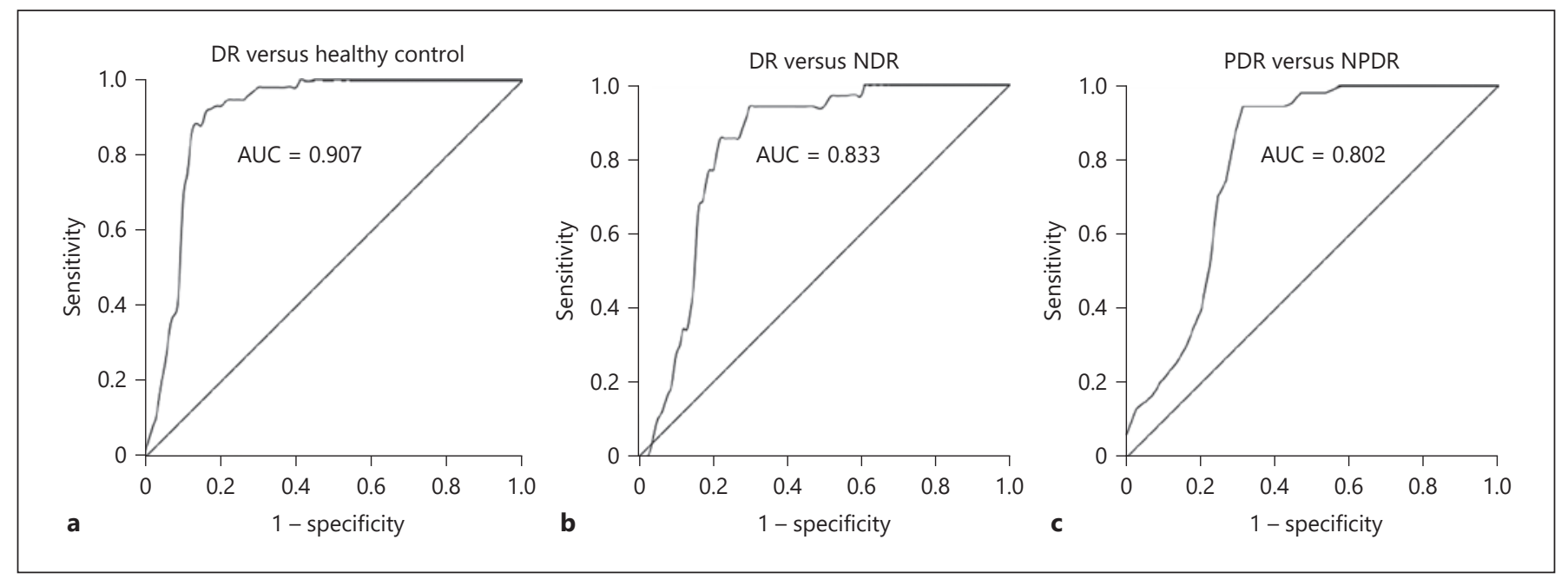

Fig. 2. ROC curve was used to evaluate the diagnostic value of miR425-5p in DR patients. a The expression of miR-425-5p can significantly distinguish healthy patients from DR patients. b Serum miR-425-5p has a high predictive value for DR patients in DM patients. c miR-425-5p has a certain diagnostic value in differenti- ating PDR patients from NPDR patients. ROC, receiver operating characteristic; DR, diabetic retinopathy; DM, diabetes mellitus; PDR, proliferative DR; NPDR, non-PDR; NDR, diabetic mellitus patients without DR; AUC, area under the curve.

\section{Results}

\section{Expression Levels of miR-425-5p in Different Patient Groups}

The serum miR-425-5p in the serum of healthy controls, NDR, NPDR, and PDR patients were first detected. As shown in Figure 1, the expression level of miR$425-5 p$ in the serum of DR patients was significantly higher than that in the healthy control group $(p<0.05)$. Also, serum miR-425-5p levels in NPDR and PDR patients were significantly higher than those in NDR patients $(p<0.05)$. More importantly, the serum miR425-5p level increased the most in PDR patients among different DR patients. The results indicated that miR425-5p may be a key biomolecule in DR and play an important biological role in the progression of the disease.

Correlation Analysis of Serum between miR-425-5p and Clinical Characteristics of DR Patients

We further investigated the correlation between the serum miR-425-5p levels and clinicopathologic characteristics in DR patients. As shown in Table 2, the expression of miR-425-5p was greatly positively correlated with the FPG, HbA1C, HOMA-IR, and disease course of the patients but showed no significant correlation with the age, sex, BMI, TG, TC, fasting insulin, and other indicators of the patients.

miR-425-5's Clinical Significance in DR Patients
Table 2. Correlation of miR-425-5p with the clinical characteristics of DR patients

\begin{tabular}{|c|c|c|}
\hline \multirow[t]{2}{*}{ Indicator } & \multicolumn{2}{|c|}{ miR-425-5p } \\
\hline & $R$ & $p$ value \\
\hline Age, years & 0.000 & 0.997 \\
\hline Gender & 0.120 & 0.233 \\
\hline $\mathrm{BMI}$ & 0.000 & 0.999 \\
\hline Disease course & 0.720 & $<0.001^{*}$ \\
\hline $\mathrm{TC}$ & 0.107 & 0.288 \\
\hline TG & 0.061 & 0.549 \\
\hline FPG & 0.799 & $<0.001^{*}$ \\
\hline $\mathrm{HbA1c}$ & 0.807 & $<0.001^{*}$ \\
\hline HOMA-IR & 0.651 & $<0.001^{*}$ \\
\hline FINS & 0.163 & 0.105 \\
\hline
\end{tabular}

TC, total cholesterol; TG, triacylglycerol; FPG, fasting plasma glucose; HbA1c, glycosylated hemoglobin; HOMA-IR, homeostasis model assessment of insulin resistance; FINS, fasting insulin.; DR, diabetic retinopathy. ${ }^{*} p<0.05$.

\section{Clinical Diagnostic Value of Serum miR-425-5p in}

Patients with DR

According to the expression level of miR-425-5p in DR patients and healthy control, ROC curve analysis was done to examine the clinical diagnostic value of serum miR-425-5p in patients of DR. The results showed 


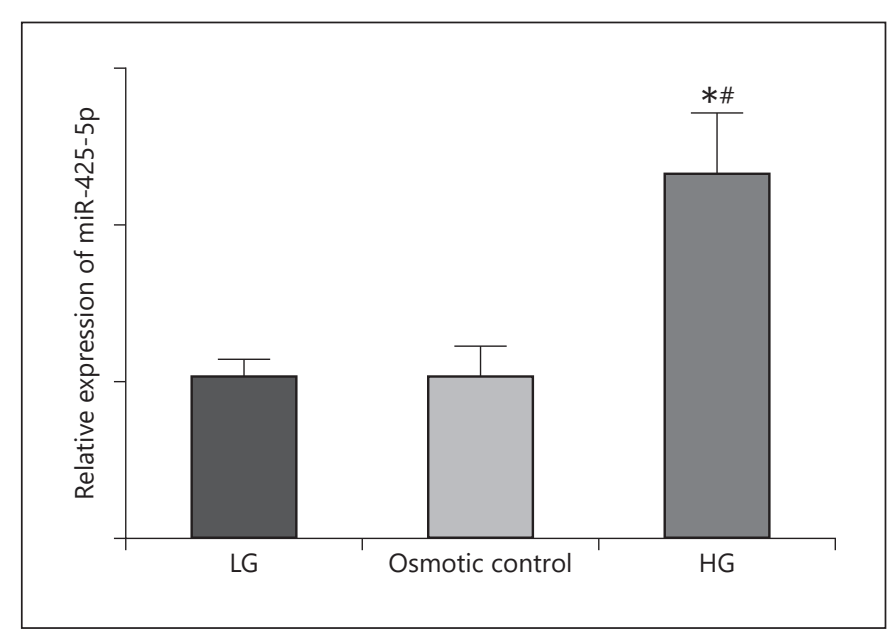

Fig. 3. Compared with LG and osmotic control, the expression level of miR-425-5p in HG-induced HRMECs was significantly increased. LG (5 mM); HG(25 mM); osmotic control (5 mM glucose +20 mM mannitol); ${ }^{*} p<0.05,5$, compared with LG group; ${ }^{*} p<$ 0.05 , compared with osmotic control. HG, high glucose; LG, low glucose; HRMECs, human retinal microvascular endothelial cells.

that the level of miR-425-5p can be used to distinguish DR patients from healthy controls, the area under the curve (AUC) was 0.907 , at the cutoff value was 1.565 , sensitivity and specificity were $91.7 \%$ and $84 \%$, respectively (Fig. 2a). Besides, the ROC curve was further drawn according to the level of the serum miR-425-5p in NDR patients and DR patients. As shown in Figure $2 \mathrm{~b}$, the AUC was 0.833 , the sensitivity was $85.7 \%$, the specificity was $78 \%$, and the cutoff value was 1.71 , indicating that serum miR-425-5p had a high predictive value for DR patients among DM patients. Finally, we further analyzed the predictive value of $\mathrm{miR}-425-5 \mathrm{p}$ for NPDR patients. It was observed that the AUC was 0.802 , the sensitivity and specificity were $94.5 \%$ and $71.1 \%$, respectively, and the cutoff value was 2.68 (Fig. 2c). The study results confirmed that miR-425-5p has a certain diagnostic value in distinguishing NPDR and PDR patients.

\section{Changes of miR-425-5p in HG-Induced HRMECs}

Changes in the miR-425-5p expression level in HRMECs under HG conditions were also detected. Results are shown in Figure 3; compared with the control group (LG, $5 \mathrm{~mm}$ glucose) and osmotic control group (5 mM glucose $+20 \mathrm{mM}$ mannitol), the expression level of miR425-5p in HRMECs was significantly increased after 25 $\mathrm{mM}$ induction $(p<0.05)$. This was consistent with the expression level of miR-425-5p observed in the serum of
DR patients. Besides, there was no significant difference between the control group and osmotic pressure control group, so LG was used as the control group in the subsequent experiments.

\section{Effects of miR-425-5p on the Proliferation and Migration of HRMECs}

As shown in Figure $4 \mathrm{a}$, the in vitro regulation of miR$425-5 p$ by transfection mimics and inhibitors was performed. The results indicated that miR-425-5p mimics can upregulate the expression level of miR-425-5p in HRMECs under HG induction, while miR-425-5p inhibitor downregulated the expression level of miR-425-5p in HRMECs under HG induction $(p<0.05)$. Cell function changes were further detected. As in previous studies, HG-induced proliferation and migration of HRMECs were promoted. However, this promotion effective was markedly reversed by an miR-425-5p inhibitor $(p<0.05$, Fig. 4b, c).

\section{Discussion}

As a chronic and serious ocular complication related to DM, DR is closely related to the pathological changes of hyperglycemia and can induce angiogenesis, inflammation, and cell proliferation [17]. Other microvascular and macrovascular complications associated with DM with diabetes also occur. At present, the effective treatment strategy for $\mathrm{DR}$ is only applicable to advanced patients, and there are obvious side effects, so it is urgent to find noninvasive and sensitive early DR diagnostic markers and for high-risk patients [18]. DRs characterized by microvascular structural and functional abnormalities and dysfunction, including retinal vascular occlusion and osmosis, which can result in nonproliferating macular edema and angiogenesis, and a larger number of highly permeable vessels during the proliferating phase [19]. The specific miRNA can destroy the blood vessel-retinal barrier and selectively secrete into the circulatory system through small membrane bubbles (such as exosomes) [20].

Previous studies have also demonstrated that specific miRNA expression patterns and levels can reveal physiological and pathological changes in patients with T2DM patients and associated microvascular and macrovascular complications, and are considered to be valuable new biomarkers [21]. For example, miR-29b is highly expressed in the plasma of DM patients and is not only correlated with the severity of DM patients, but also with the clinicopathologic of the patients [22]. MiR-217 is upregulated 


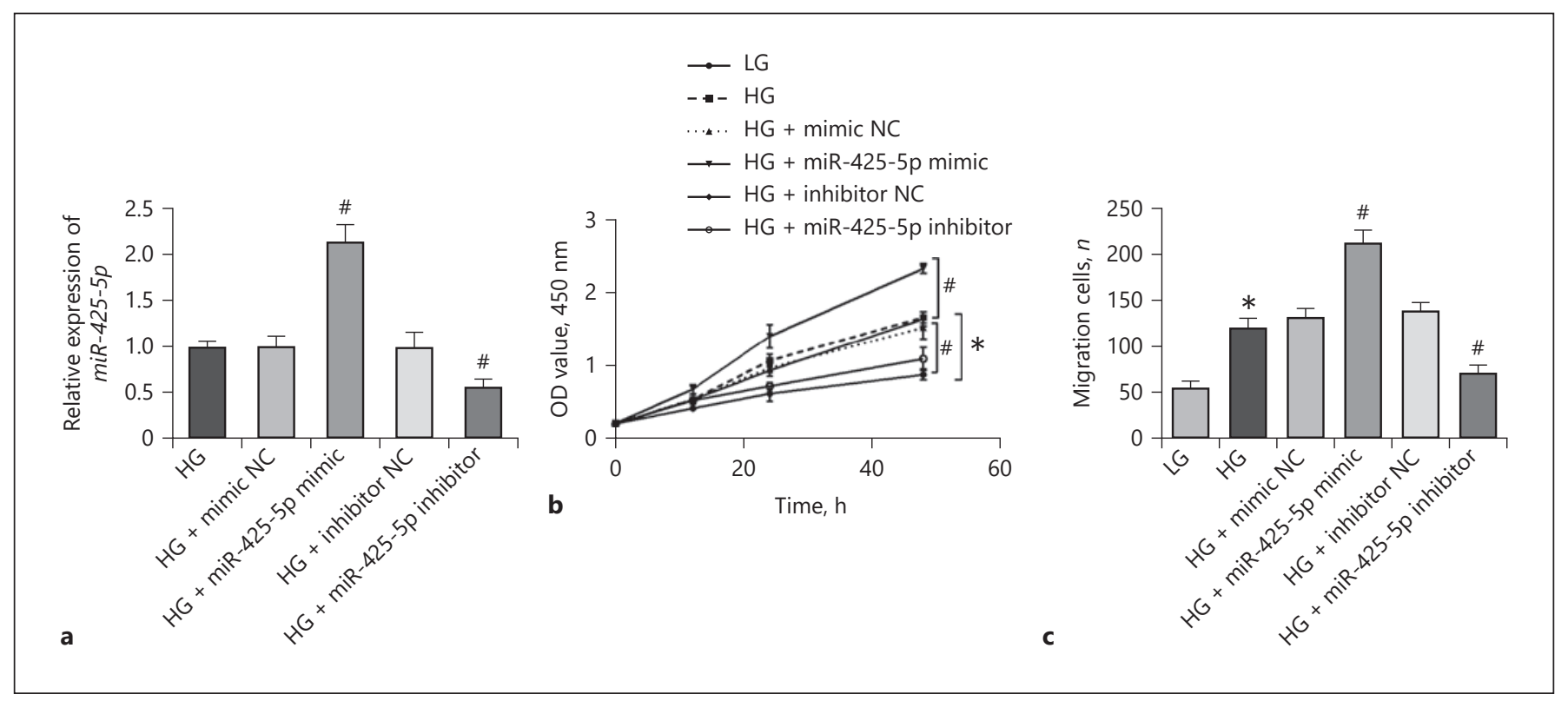

Fig. 4. Effects of in vitro regulation of miR-425-5p on cell proliferation and migration under HG conditions. a Changes in expression levels of miR-425-5p after transfection with miR-425-5p mimic and inhibitor. $\mathbf{b}$ Changes in the proliferation of miR-425-5p

in human retinal pigment epithelial cells induced by HG can be used as a biomarker for DR [23]. Notably, a recent study found that miR-425-5p was significantly upregulated in plasma and peripheral blood mononuclear cells of $30 \mathrm{DM}$ patients, and was involved in vascular endothelial dysfunction in DM [11]. Additionally, Shao et al. [10] have found that miR-425-5p is upregulated in the serum of DR patients via a microarray analysis. In this study, compared with the healthy control group, the expression level of miR-425-5p in the serum of DR patients was significantly increased, which was consistent with the previous evidence. Meanwhile, the expression level of miR425-5p in the serum of PDR patients was significantly higher than that of NDR and NPDR patients. The results suggested that miR-425-5p may be involved in the development of DR.

To further confirm the potential mechanism of miR425-5p in DR, we studied the correlation between the serum miR-425-5p level and the clinical characteristics of DR patients. The American Diabetes Association recommends an FPG of $7.0 \mathrm{mmol} / \mathrm{L}$ as a threshold for diagnosing the presence of DM [24,25], and its levels can be used to predict DR [26]. HbA1c is a routine standard for long-term blood glucose control, which can predict DM patients and their complications. What is more, the increase in $\mathrm{HbAlc}$ can shorten the occurrence time and increase the occurrence fre-

miR-425-5's Clinical Significance in DR Patients cells under HG conditions. c Effect of miR-425-5p on cell migration under HG conditions. LG: 5 mM; HG: 25 mM; ${ }^{*} p<0.05$. Compared with the LG group; ${ }^{\sharp} p<0.05$ compared to the HG group. HG, high glucose; LG, low glucose.

quency of DR lesions [27, 28]. HOMA-IR is used to assess insulin resistance, and the increase in HOMA-IR is accustomed to evaluate the progression of DM [29]. Therefore, in our research, the correlations of serum miR-425-5p with FPG, HbAlc, and HOMA-IR were evaluated, and the results confirmed that serum miR-425-5p was a large extent positively correlated with them. The results showed that miR-425-5p serves a critical role in DR development.

Previous studies have shown that the disorder of miR$425-5 p$ can be used to diagnose a variety of certain diseases, such as plasma miR-425-5p can be used as a new noninvasive biomarker for diagnosing colorectal cancer [30]. miR-425-5p has certain specificity and sensitivity and is a valuable diagnostic marker for early gastric cancer [31]. Therefore, in this study, we used the ROC curve to test the diagnostic value of miR-425-5p in DR patients. Our results confirmed that serum miR-425-5p can not only distinguish DR patients from healthy individuals and DM patients but also distinguish PDR patients from NPDR patients. Our results indicate that miR-425-5p is a critical biological diagnostic marker in DR patients and can predict the development and severity of DR.

Pathological angiogenesis and abnormal proliferation are some of the reasons leading to the occurrence of retinal angiopathy and severe visual impairment. Therefore, it is very important to develop methods of inhibiting an- 
giogenesis to prevent the occurrence of DR [32]. The regulation of hyperglycemia-induced angiogenesis has been used for the prevention and treatment of DR [33]. Gao et al. [34] studied that miR-425-5p plays a key regulatory role in arsenic-induced angiogenesis. HRMECs have been widely used in DR's research on endothelial cell function. Our study showed that HG-induced HRMECs were used to study the effect of miR-425-5p on the function of vascular endothelial cells. The results showed that compared with the control group and osmotic group, HG induction can lead to an increase in the level of miR-425$5 p$ in HRMECs. Under HG conditions, overexpression of miR-425-5p promoted HRMEC proliferation and migration, while inhibition of miR-425-5p led to opposite results. The experimental results confirmed that miR-425$5 p$ may play important regulatory roles in the formation of new blood vessels in the pathogenesis of DR. Our study has some limitations, and the target gene of miR-425-5p in DR and its specific mechanism still need further study.

The main limitation of the current study is that the sample size is relatively small, which may have a certain impact on the statistical ability, such as there was no statistical difference in the indicators such as TC, and TG. Besides, the target genes of miR-425-5p in DR have not been studied, we will focus on them in the following.

Taken together, the research confirmed that the serum miR-425-5p was elevated in DR patients. Moreover, it can be used as an effective biomarker for the diagnosis of DR patients and can predict the development and severity of DR. Moreover, inhibiting the expression of miR-425-5p under the condition of hyperglycemia can be used as a potential therapeutic strategy to inhibit the pathogenesis of DR.

\section{Statement of Ethics}

The research methods meet the standards set out in the Helsinki Declaration. And the study was approved by the Research Ethics Committee of the Affiliated Hospital of Weifang Medical University (No. 2015-097). All participants were informed of the purpose of the study and experimental procedures and signed a consent form approved by the Ethics Committee before participating.

\section{Conflict of Interest Statement}

The authors have no conflicts of interest to declare.

\section{Funding Sources}

This study did not receive any funding.

\section{Author Contributions}

All authors contributed to the study conception and design. Material preparation, data collection, and analysis were performed by Xia Liu, Yanhua Zhou and Yunxia Liu. The first draft of the manuscript was written by Xia Liu, and all authors commented on previous versions of the manuscript. All authors read and approved the final manuscript.

\section{Data Availability Statement}

All data generated or analyzed during this study are included in this article. Further enquiries can be directed to the corresponding author.

\section{References}

1 Yang $\mathrm{H}$, Gan S, Jiang Z, Song X, Chen T, Xu $Y$, et al. Protective effects of essential oil from fructus alpiniae zerumbet on retinal müller gliosis via the PPAR- $\gamma$-p-CREB signaling pathway. Chin Med. 2020;15:4.

2 Shao Y, Dong LJ, Takahashi Y, Chen J, Liu X, Chen $\mathrm{Q}$, et al. miRNA-451a regulates RPE function through promoting mitochondrial function in proliferative diabetic retinopathy. Am J Physiol Endocrinol Metab. 2019 Mar 1; 316(3):E443-E52.

3 Bao XY, Cao J. MiRNA-138-5p protects the early diabetic retinopathy by regulating NOVA1. Eur Rev Med Pharmacol Sci. 2019 Sep;23(18):7749-56.

4 de Carlo TE, Bonini Filho MA, Baumal CR, Reichel E, Rogers A, Witkin AJ, et al. Evaluation of preretinal neovascularization in proliferative diabetic retinopathy using optical co- herence tomography angiography. Ophthalmic Surg Lasers Imaging Retina. 2016 Feb; 47(2):115-9.

5 Murata M, Noda K, Ishida S. Pathological role of unsaturated aldehyde acrolein in diabetic retinopathy. Front Immunol. 2020;11:589531.

6 Lopez-Contreras AK, Martinez-Ruiz MG, Olvera-Montano C, Robles-Rivera RR, ArevaloSimental DE, Castellanos-Gonzalez JA, et al. Importance of the use of oxidative stress biomarkers and inflammatory profile in aqueous and vitreous humor in diabetic retinopathy. Antioxidants. 2020 Sep 20;9(9):891.

7 Haque MM, Murale DP, Lee JS. Role of microRNA and oxidative stress in influenza a virus pathogenesis. Int J Mol Sci. 2020 Nov 25; 21(23):8962.

8 Guan JT, Li XX, Peng DW, Zhang WM, Qu J, Lu F, et al. MicroRNA-18a-5p administration suppresses retinal neovascularization by targeting FGF1 and HIF1A. Front Pharmacol. 2020;11:276.

9 Prado MSG, de Goes TC, de Jesus ML, Mendonça LSO, Nascimento JS, Kaneto CM. Identification of miR-328-3p as an endogenous reference gene for the normalization of miRNA expression data from patients with diabetic retinopathy. Sci Rep. 2019 Dec 23;9(1):19677.

10 Shao J, Fan G, Yin X, Gu Y, Wang X, Xin Y, et al. A novel transthyretin/STAT4/miR-2233p/FBXW7 signaling pathway affects neovascularization in diabetic retinopathy. Mol Cell Endocrinol. 2019 Dec 1;498:110541.

11 Luo E, Wang D, Yan G, Qiao Y, Zhu B, Liu B, et al. The NF-kappaB/miR-425-5p/MCT4 axis: a novel insight into diabetes-induced endothelial dysfunction. Mol Cell Endocrinol. 2020 Jan 15;500:110641. 
12 American Diabetes A. Executive summary: standards of medical care in diabetes: 2014. Diabetes Care. 2014 Jan;37(Suppl 1):S5-13.

13 American Diabetes A. 2. Classification and diagnosis of diabetes: standards of medical care in diabetes-2019. Diabetes Care. 2019 Jan;42(Suppl 1):S13-28.

14 Prado MSG, de Jesus ML, de Goes TC, Mendonça LSO, Kaneto CM. Downregulation of circulating miR-320a and target gene prediction in patients with diabetic retinopathy. BMC Res Notes. 2020 Mar 16;13(1):155.

15 Platania CBM, Pittalà V, Pascale A, Marchesi $\mathrm{N}$, Anfuso CD, Lupo G, et al. Novel indole derivatives targeting HuR-mRNA complex to counteract high glucose damage in retinal endothelial cells. Biochem Pharmacol. 2020 May; $175: 113908$.

16 Zeng Q, Liu J. Silencing circ_0001879 inhibits the proliferation and migration of human retinal microvascular endothelial cells under high-glucose conditions via modulating miR30-3p. Gene. 2020 Nov 15;760:144992.

17 Li P, Yan X, Xu G, Pang Z, Weng J, Yin J, et al. A novel plasmalncRNAENST00000416361 is upregulated in coronary artery disease and is related to inflammation and lipid metabolism. Mol Med Rep. 2020 Jun;21(6):2375-84.

18 Kubota R, Jhaveri C, Koester JM, Gregory JK Effects of emixustat hydrochloride in patients with proliferative diabetic retinopathy: a randomized, placebo-controlled phase 2 study. Graefes Arch Clin Exp Ophthalmol. $2021 \mathrm{Feb}$ 259(2):369-78.

19 Liu HN, Li X, Wu N, Tong MM, Chen S, Zhu SS, et al. Serum microRNA-221 as a biomarker for diabetic retinopathy in patients associated with type 2 diabetes. Int J Ophthalmol. 2018;11(12):1889-94.
20 La Marca V, Fierabracci A. Insights into the diagnostic potential of extracellular vesicles and their miRNA signature from liquid biopsy as early biomarkers of diabetic micro/macrovascular complications. Int J Mol Sci. 2017 Sep 14;18(9): 1974

21 Liu HN, Cao NJ, Li X, Qian W, Chen XL. Serum microRNA-211 as a biomarker for diabetic retinopathy via modulating Sirtuin 1 . Biochem Biophys Res Commun. 2018 Nov 10;505(4):1236-43.

22 Alfaifi M, Verma AK, Alshahrani MY, Joshi PC, Alkhathami AG, Ahmad I, et al. Assessment of cell-free long non-coding RNA-H19 and miRNA-29a, miRNA-29b expression and severity of diabetes. Diabetes Metab Syndr Obes. 2020;13:3727-37.

23 Xiao H, Liu Z. Effects of microRNA217 on high glucoseinduced inflammation and apoptosis of human retinal pigment epithelial cells (ARPE19) and its underlying mechanism. Mol Med Rep. 2019 Dec;20(6):5125-33.

24 Wong TY, Liew G, Tapp RJ, Schmidt MI, Wang JJ, Mitchell P, et al. Relation between fasting glucose and retinopathy for diagnosis of diabetes: three population-based cross-sectional studies. Lancet. 2008 Mar 1;371(9614): 736-43.

25 Lailler G, Piffaretti C, Fuentes S, Nabe HD, Oleko A, Cosson E, et al. Prevalence of prediabetes and undiagnosed type 2 diabetes in France: results from the national survey ESTEBAN, 2014-2016. Diabetes Res Clin Pract. 2020 Jul;165:108252.

26 Takao $\mathrm{T}$, Inoue $\mathrm{K}$, Suka $\mathrm{M}$, Yanagisawa $\mathrm{H}$, Iwamoto Y. Optimal cutoff values of fasting plasma glucose (FPG) variability for detecting retinopathy and the threshold of FPG levels for predicting the risk of retinopathy in type 2 diabetes: a longitudinal study over 27years. Diabetes Res Clin Pract. 2018 Jun; 140:22835.
27 Chamberlain JJ, Herman WH, Leal S, Rhinehart AS, Shubrook JH, Skolnik N, et al. Pharmacologic therapy for type 2 diabetes: synopsis of the 2017 American diabetes association standards of medical care in diabetes. Ann Intern Med. 2017 Apr 18;166(8):572-8.

28 Andreasson R, Ekelund C, Landin-Olsson M, Nilsson C. HbA1c levels in children with type 1 diabetes and correlation to diabetic retinopathy. J Pediatr Endocrinol Metab. 2018 Mar 28;31(4):369-74.

29 Morimoto A, Tatsumi Y, Soyano F, Miyamatsu N, Sonoda N, Godai K, et al. Increase in homeostasis model assessment of insulin resistance (HOMA-IR) had a strong impact on the development of type 2 diabetes in Japanese individuals with impaired insulin secretion: the Saku study. PLoS One. 2014;9(8): e105827.

30 Tan Y, Lin JJ, Yang X, Gou DM, Fu L, Li FR, et al. A panel of three plasma microRNAs for colorectal cancer diagnosis. Cancer Epidemiol. 2019 Jun;60:67-76.

31 Zhu XL, Ren LF, Wang HP, Bai ZT, Zhang L, Meng WB, et al. Plasma microRNAs as potential new biomarkers for early detection of early gastric cancer. World J Gastroenterol. 2019 Apr 7;25(13):1580-91.

32 Matos AL, Bruno DF, Ambrósio AF, Santos PF. The benefits of flavonoids in diabetic retinopathy. Nutrients. 2020 Oct 16;12(10).

33 Anitha RE, Janani R, Peethambaran D, Baskaran V. Lactucaxanthin protects retinal pigment epithelium from hyperglycemia-regulated hypoxia/ER stress/VEGF pathway mediated angiogenesis in ARPE-19 cell and rat model. Eur J Pharmacol. 2021 Mar 8;899: 174014.

34 Gao Y, Yin Y, Xing X, Zhao Z, Lu Y, Sun Y, et al. Arsenic-induced anti-angiogenesis via miR-425-5p-regulated CCM3. Toxicol Lett. 2016 Jul 8;254:22-31. 